\title{
Numerical Simulation of Blast Wave Due to Meteorite Explosion in Stratified Atmosphere*
}

\author{
Rei YAMASHITA ${ }^{\dagger}$ and Kojiro SUZUKI \\ Department of Advanced Energy, The University of Tokyo, Kashiwa, Chiba 277-8561, Japan
}

\begin{abstract}
This paper investigates the applicability of direct simulation considering a stratified atmosphere to an unsteady analysis of a blast wave caused by a meteorite explosion. Axi-symmetric Euler equations with a gravity term that considers a horizontally stratified atmosphere are numerically solved to analyze blast wave propagation from the source of meteorite explosion to the ground. Computations are made to reproduce the blast wave generated by the Chelyabinsk meteorite in 2013, assuming a spherically uniform high-pressure and high-temperature core as the source of explosion. The amount of energy released by the explosion at an altitude of $25 \mathrm{~km}$ is assumed to be $500 \mathrm{kt}$ of trinitrotoluene, and the computational domain ranges over a radial distance of $50 \mathrm{~km}$ from the center of the explosion. The simulation results in a uniform atmosphere agree well with the results of well-known Brode's empirical formula, and the blast wave obtained from the simulation considering a standard atmosphere is expected to cause serious damage to the ground, as experienced at the time of the Chelyabinsk event. Moreover, the simulation results clarify the characteristics of blast wave propagation through uniform, isothermal, and standard atmospheres.
\end{abstract}

Key Words: Blast Wave, Meteorite, Direct Simulation, CFD

\author{
Nomenclature \\ $c$ : speed of sound \\ $e$ : total energy \\ $E_{\mathrm{TNT}}$ : TNT-equivalent energy \\ $E, F$ : flux vector in $x$ and $r$ coordinates \\ $g$ : acceleration of gravity, $9.80665 \mathrm{~m} / \mathrm{s}^{2}$ \\ $h$ : altitude \\ $h_{\mathrm{s}}$ : scale height \\ $H$ : axi-symmetric term \\ $p$ : pressure \\ $Q$ : conservative variable \\ $r$ : radial coordinate \\ $R$ : spherical radius \\ $R_{\text {air }}$ : gas constant, for air $287 \mathrm{~J} /(\mathrm{K} \cdot \mathrm{kg})$ \\ $S_{C}$ : correction term \\ $S_{G}$ : gravity term \\ $t$ : time \\ $T$ : temperature \\ $u, v$ : velocity in $x$ and $r$ coordinates \\ $u_{s}$ : propagation speed of shock wave \\ $V$ : volume of core \\ $x$ : vertical coordinate \\ $Z$ : scaled distance \\ $\beta$ : atmospheric temperature lapse rate \\ $\gamma$ : ratio of specific heat, for air 1.4 \\ $\Delta p_{s}$ : pressure rise of shock wave \\ $\rho$ : density
}

(C) 2018 The Japan Society for Aeronautical and Space Sciences

*Presented at the 60th Space Science and Technology Conference, 6-9 September 2016, Hakodate, Japan.

Received 17 October 2017; accepted for publication 22 February 2018.

†Corresponding author, yamashita@daedalus.k.u-tokyo.ac.jp $\theta$ : rotational coordinate

Subscripts

0 : reference value at ground

core: explosive core

$\infty$ : atmosphere

\section{Introduction}

Meteorites have been observed an average of six times per year in the past century, and natural disasters due to the meteorites occurred about once a decade. ${ }^{1)}$ Generally, the effects of a meteorite depend on its size, and it has been found that a disaster occurs when meteorites are larger than $10 \mathrm{~m}$ in diameter. ${ }^{2)}$ The Chelyabinsk meteorite observed in 2013 was assumed to have a diameter of $19 \mathrm{~m} .{ }^{3,4)}$ Since this meteorite exploded during atmospheric entry, small fragments generated by the explosion fell to the ground, and the blast wave generated from the explosion propagated toward the ground. Consequently, the blast wave, rather than the impact of the fragments, injured more than 1,000 people and damaged many buildings throughout the city of Chelyabinsk. The largest meteorite in the 20th century was $30 \mathrm{~m}$ in diameter and was observed in the Tunguska river area. ${ }^{5)}$ Although this meteorite burned up over the ground, the blast wave generated from the explosion caused extensive tree damage over an area of $2,000 \mathrm{~km}^{2}$. These facts indicate that natural disasters caused by meteorites are caused by the blast waves rather than the meteorite impact, and the risk management of natural disasters should be conducted. Thus, studies on planetary defense ${ }^{6)}$ have been carried out, particularly after the Chelyabinsk event. For risk management, ${ }^{1)}$ meteorites must be detected in advance, and an evacuation guideline for meteorites must be developed based on strength of the blast wave, which 
depends on the explosive conditions, including the explosive energy, altitude of explosion, and atmospheric conditions. Therefore, parametric studies for meteorite risk management, ${ }^{6}$ ) which has not been conducted to date, are required.

Blast wave predictions have been conducted using TNTequivalent methods, ${ }^{7,8)}$ but these fail to properly consider effects such as atmospheric stratification, thermochemical nonequilibrium, and ground effects. Hence, an accurate prediction method considering these effects should be developed for accurately evaluating the influence of meteorite fall. Thus far, such methods ${ }^{9,10)}$ have been developed based on the weak shock theory, ${ }^{11)}$ wherein a weak shock wave with $\Delta p<100 \mathrm{~Pa}$ is treated as a sound wave. Although prediction methods have been used for evaluating sonic boom phenomenon ${ }^{9)}$ caused by shock waves created by supersonic aircraft, they cannot be applied to analysis of blast waves caused by meteorite explosion for the following reason. The assumption of a weak shock wave is not applicable until the blast wave moves far away from the center of explosion; i.e., the prediction methods for blast wave must be applicable even to $\Delta p>100 \mathrm{~Pa}$. One promising candidate to accurately analyze not only weak shock waves, but also strong shock waves is a direct simulation based on Computational Fluid Dynamics (CFD), wherein the computational domain ranges from the generation source of the shock wave to observation points. The direct simulation of a blast wave has been performed to investigate the influence of volcanic eruptions, ${ }^{12}$ ) rocket launches in the vicinity of the launching site, ${ }^{13)}$ and the risk assessment of hydrogen application. ${ }^{14)}$ In addition, a simulation has been recently performed to evaluate the blast wave at the time of the Chelyabinsk meteorite. ${ }^{6,15)}$ However, it has been difficult to consider a realistic stratified atmosphere in a simulation for the following reason: The change in atmospheric pressure with altitude is assumed to be a discontinuity in the Riemann solver, which is often used for compressible CFD analysis, and the hydrostatic balance between gas pressure and gravitational force cannot be strictly maintained. ${ }^{16)}$ For this reason, simplified atmospheric models such as uniform atmospheres and isothermal atmospheres $^{6)}$ have been used for direct simulation, although the difference in blast wave propagation between the real atmospheres and the simplified atmospheres has not yet been investigated. Thus, a simulation method considering real atmosphere is required to accurately evaluate the strength of the blast wave, and atmospheric effects should be investigated in detail.

Direct simulations have been performed not only to analyze blast waves, but also to analyze sonic booms. The latest simulation for a sonic boom was performed using a numerical correction method ${ }^{17)}$ to strictly maintain hydrostatic balance in the Riemann solver, and the simulation results were in good agreement with the observation. Although the direct simulation of sonic booms has been performed under the assumption of steady-state flow, the simulation method is expected to be a powerful tool for the unsteady analysis of blast waves, which is required to enable the risk management of meteorites. In addition, the simulation will be helpful in in- vestigating the effect of atmospheric stratification, which has not been well clarified. With this in mind, the objectives of this study are to develop a direct simulation method that considers a stratified atmosphere for the unsteady analysis of the blast wave generated by a meteorite explosion and to clarify the effect of atmospheric stratification. In particular, the application of direct simulation for unsteady analysis of the blast wave in a uniform atmosphere is validated by comparison with Brode's empirical formula, ${ }^{8)}$ which is one of the most famous TNT-equivalent methods. Thereafter, computations are made to reproduce the blast wave generated by the Chelyabinsk meteorite in 2013, and the simulation results are validated by comparison with observed facts. Moreover, the simulation results are analyzed to clarify the nature of blast wave propagation through a stratified atmosphere.

\section{Computational Conditions}

\subsection{Modeling of meteorite explosion}

The Chelyabinsk meteorite ${ }^{3,4)}$ was observed in many videos and observation equipment, and the circumstances of the meteorite fall are well understood based on the observational data. Thus, computations were made to reproduce the blast wave generated by the Chelyabinsk meteorite in 2013. Figure 1 shows an outline of computational conditions. Since the meteorite explosion occurred during atmospheric entry, it should be modeled as a moving source along a trajectory. However, the drop speed of the meteorite was much faster than the propagation speed of the blast wave, and thus the explosion is assumed to have occurred instantaneously. In addition, a previous study ${ }^{6}$ confirmed that the strength of the blast wave generated from a cylindrical source is almost the same as that generated from a spherical source with the same level of explosive energy in an area far away from ground zero, i.e., the point on the ground vertically below the center of the explosion shown in Fig. 1. Since the city of Chelyabinsk was approximately $40 \mathrm{~km}$ from ground zero, the assumption of a spherical source was convenient not only for the simplicity of analysis, but also for reducing the computational cost. Hence, a spherically uniform highpressure and high-temperature core was assumed as the
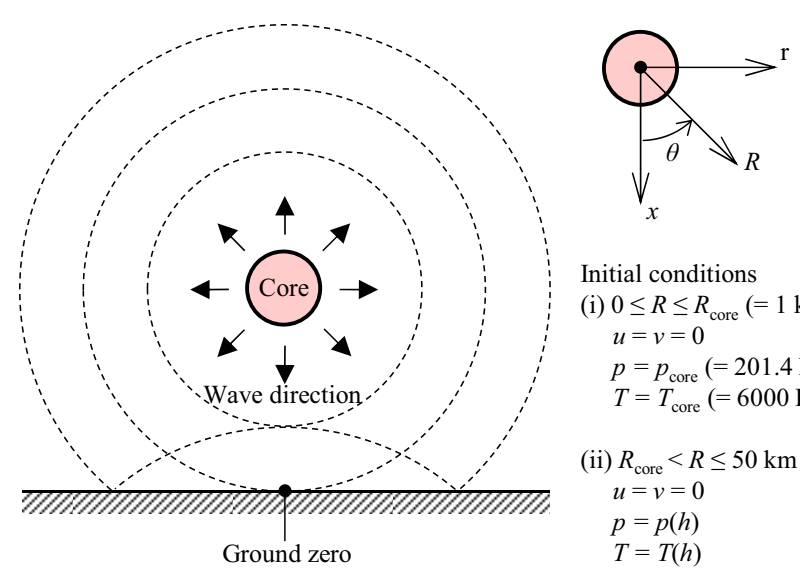

$$
\begin{aligned}
& \text { Initial conditions } \\
& \text { (i) } 0 \leq R \leq R_{\text {core }}(=1 \mathrm{~km}) \\
& u=v=0 \\
& p=p_{\text {core }}(=201.4 \mathrm{kPa}) \\
& T=T_{\text {core }}(=6000 \mathrm{~K}) \\
& \text { (ii) } R_{\text {core }}<R \leq 50 \mathrm{~km} \\
& u=v=0 \\
& p=p(h) \\
& T=T(h)
\end{aligned}
$$

Fig. 1. Outline of computational conditions. 
source of meteorite explosion. Note that the assumption of a spherical core is used in the analysis of the meteorite explosion due to the fact that the source of the meteorite explosion was well modeled by the core. ${ }^{6}$ As shown in Ref. 3), the amount of energy released by the explosion at an altitude of $25 \mathrm{~km}$ was assumed to be $E_{\mathrm{TNT}}=500 \mathrm{kt}$ of trinitrotoluene, and the uniform temperature in the core was set to $T_{\text {core }}=6,000 \mathrm{~K}$. The core radius was set to $R_{\text {core }}=1 \mathrm{~km}$, corresponding to the size of fireball observed in Chelyabinsk. Under the above conditions, the uniform pressure in the core was calculated using Brode's equation, ${ }^{8)}$ given by Eq. (1), which is often used to predict the explosive energy generated by compressed gas.

$$
\begin{gathered}
E_{\mathrm{TNT}}=\frac{p_{\text {core }}-p_{\infty, \text { core }}}{\gamma-1} V \\
V=\frac{4}{3} \pi R_{\text {core }}{ }^{3}
\end{gathered}
$$

As a result, the uniform pressure in the core was $p_{\text {core }}=$ $201.4 \mathrm{kPa}$, where the atmospheric pressure at $h=25 \mathrm{~km}$ was $p_{\infty, \text { core }}=1.596 \mathrm{kPa}$. The computational domain ranges over a radial distance of $50 \mathrm{~km}$ from the center of the explosion, and the propagation time of the blast wave was set to $150 \mathrm{~s}$.

\subsection{Atmospheric models}

The standard atmospheric condition was set based on the ISO standard atmosphere ${ }^{18)}$ as follows. The atmospheric temperature is defined using Eq. (3), and the analytical solutions of atmospheric pressure and density are calculated using the equation of the state of ideal gas given by Eq. (4) and the hydrostatic equation given by Eq. (5).

$$
\begin{gathered}
T_{\infty}=T_{0}-\int_{0}^{h} \beta d h \\
p_{\infty}=\rho_{\infty} R_{\text {air }} T_{\infty} \\
\frac{d p_{\infty}}{d h}=-g \rho_{\infty}
\end{gathered}
$$

Since the Chelyabinsk meteorite was observed in February 2013, the atmospheric pressure and temperature at groundlevel were set to $T_{0}=263.15 \mathrm{~K}$ and $p_{0}=101.325 \mathrm{kPa}$, respectively. The temperature lapse rate $\beta$ was the same as that of the ISO standard atmosphere. Figure 2 shows the variation in atmospheric temperature and pressure with altitude. To investigate the effect of atmospheric stratification, simulations were performed not only for standard atmosphere, but also for isothermal and uniform atmospheres, which have been used for the analysis of blast waves. ${ }^{6)}$ The atmospheric properties of the isothermal atmosphere were set as

$$
\begin{gathered}
\frac{p_{\infty}}{p_{0}}=e^{-h / h_{\mathrm{s}}} \\
T_{\infty}=\frac{g h_{\mathrm{s}}}{R_{\mathrm{air}}}
\end{gathered}
$$

where the scale height $h_{\mathrm{s}}$ was set as

$$
h_{\mathrm{s}}=\frac{h}{\ln \left(p_{\infty, \text { core }} / p_{0}\right)}
$$
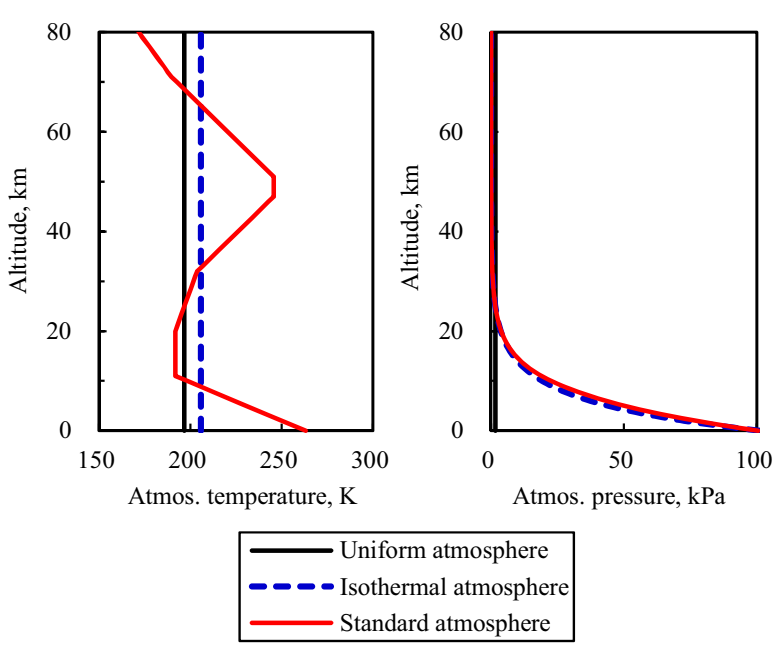

Fig. 2. Atmospheric condition.

The pressure of the isothermal atmosphere corresponds to that of the standard atmosphere at both ground-level and the center of the explosion, although the rate of change in atmospheric pressure differs in the isothermal and standard atmospheres. The atmospheric properties of the uniform atmosphere were set to those of the standard atmosphere at an altitude of $25 \mathrm{~km}$. Note that the pressure rise of the blast wave and its propagation speed depend on the atmospheric pressure and temperature, respectively. Thus, effects due to change in the atmospheric pressure and temperature can be investigated by comparing the three atmospheric models.

\section{Computational Method}

\subsection{Governing equations}

The blast wave generated from a spherical core propagates spherically. However, the characteristics of the blast wave propagation change according to the propagation direction because of atmospheric stratification with altitude. Thus, a spherical symmetry cannot be assumed, and instead, the flow field was assumed to be axi-symmetric with respect to the $x$-axis shown in Fig. 1. The governing equations were set to axi-symmetric Euler equations with a gravity term to consider the horizontally stratified atmosphere:

$$
\frac{\partial Q}{\partial t}+\frac{\partial E}{\partial x}+\frac{\partial F}{\partial r}+H=S_{G}
$$

$$
\begin{aligned}
& Q=\left[\begin{array}{c}
\rho \\
\rho u \\
\rho v \\
e
\end{array}\right], \quad E=\left[\begin{array}{c}
\rho u \\
\rho u^{2}+p \\
\rho u v \\
(e+p) u
\end{array}\right], \quad F=\left[\begin{array}{c}
\rho v \\
\rho u v \\
\rho v^{2}+p \\
(e+p) v
\end{array}\right], \\
& H=\left[\begin{array}{c}
\rho v / r \\
\rho u v / r \\
\rho v^{2} / r \\
(e+p) v / r
\end{array}\right], \quad S_{G}=\left[\begin{array}{c}
0 \\
\rho g \\
0 \\
\rho g u
\end{array}\right]
\end{aligned}
$$




$$
e=\frac{\rho R_{\mathrm{air}} T}{\gamma-1}+\frac{\rho}{2}\left(u^{2}+v^{2}\right)
$$

The governing equations were discretized using the finite volume method, and the Riemann solver was used to accurately capture the blast waves. The inviscid terms were computed using the AUSM-DV scheme, ${ }^{19)}$ which is extended to third-order accuracy by applying Monotone Upstream-centered Schemes for Conservation Law (MUSCL) interpolation $^{20)}$ with the van Albada slope limiter. ${ }^{21)}$ Time integration was performed using the second-order Runge-Kutta method. Note that the change in atmospheric properties with altitude was treated as a discontinuity in the Riemann solver; that is, the hydrostatic balance cannot be strictly maintained and nonphysical waves are generated. To resolve this problem, the numerical correction method ${ }^{17)}$ that was used in the direct simulation of sonic booms was used with a slight modification. The correction term $S_{C}$ was artificially introduced to conserve the stationary air condition in the stratified atmosphere, where $u=v=0, T=T_{\infty}(h), p=p_{\infty}(h)$, as follows.

$$
\left(\frac{\partial Q}{\partial t}+\frac{\partial E}{\partial x}+\frac{\partial F}{\partial r}+H\right)_{i, j}=\left(S_{G}+S_{C}\right)_{i, j}
$$

The correction term was evaluated according to the following procedure:

1) The stationary air condition in the stratified atmosphere was set in the entire computational domain including the entire boundaries.

2) The numerical fluxes were calculated and the correction term was calculated as

$$
S_{C, i, j}=\left(\frac{\partial E}{\partial x}+\frac{\partial F}{\partial r}+H-S_{G}\right)_{i, j}
$$

3) The correction term obtained in step 2) was used as the constant value in the simulation.

Using the numerical correction method above, the fluctuation components from the stationary air condition in a stratified atmosphere were calculated.

\subsection{Computational grid}

Figure 3 shows the computational grid and the computational domain. The computational grid was a semicircular shape that extended from $R=0$ to $50 \mathrm{~km}$. To consider the effects of ground reflection, the grid points were located on the ground surface at an altitude of $0 \mathrm{~km}$, and the region under the surface was not computed. The grid spacing in the $R$ direction was constant and that in the $\theta$ direction was changed according to distance from the center of explosion. To confirm the grid convergence, simulations for standard atmosphere were performed using coarse $(2,501 \times 101)$ and fine $(5,001 \times 201)$ grids. They took approximately 3.5 hours using the coarse grid and 45.1 hours using the fine grid, and were processed using an Intel Core ${ }^{\mathrm{TM}}$ i7-5820K@3.30 $\mathrm{GHz}$ (six cores). In the results of the fine grid relative to those of the coarse grid, the pressure rise of the blast wave on the ground increased by $4.8 \%$ at $r=0 \mathrm{~km}$ and $9.0 \%$ at $r=$ $40 \mathrm{~km}$, which corresponds to distance from ground zero to
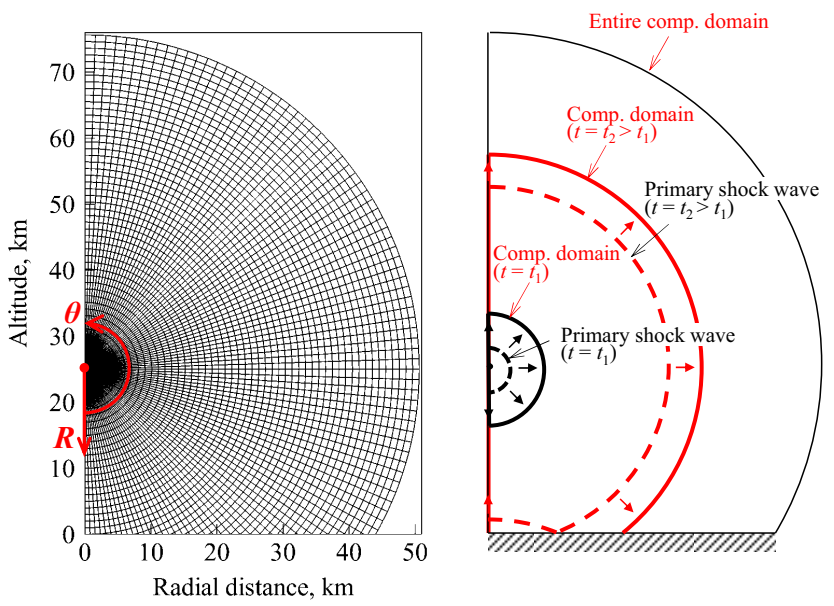

Fig. 3. Fine grid (shown every one-hundred points in the $R$ direction and every other point in the $\theta$ direction) and change of computational domain.

Chelyabinsk. Moreover, because the pressure waveforms were in good agreement with each other, the strength of the blast wave seems to be almost the same regardless of the number of grid points and grid spacing.

The computational efficiency was improved by extending the computational domain in accordance with the location of the primary shock wave, as shown in Fig. 3. In particular, the location of the primary shock wave propagating straight upward was identical to that of the peak pressure rise. Then, the computational domain in each time step was set from the center of the explosion to a location $0.5 \mathrm{~km}$ from that of the primary shock wave. At the start of computation, the computational domain was set in the region $R=0$ to $1.5 \mathrm{~km}$ and was extended gradually until $R=0$ to $50 \mathrm{~km}$.

\subsection{Boundary conditions}

The boundary conditions must be set considering not only the fluctuation of waves, but also the change in atmospheric properties with altitude. Thus, they were set as follows:

- Axial symmetry condition on the $x$-axis $(r=0)$

$$
\begin{gathered}
Q_{j=1}=Q_{j=2}, \quad \text { but } \quad v_{j=1}=0 \\
Q_{j=j \max }=Q_{j=j \max -1}, \quad \text { but } \quad v_{j=j \max }=0
\end{gathered}
$$

- Extrapolation condition at singularity $(R=0)$

$$
\begin{gathered}
u_{i=1}=v_{i=1}=0 \\
p_{i=1}=p_{\infty, \text { core }}+\Delta p_{i=2}, \quad T_{i=1}=T_{\infty, \text { core }}+\Delta T_{i=2}
\end{gathered}
$$

- Stationary air condition $\left(R=R_{\max }=50 \mathrm{~km}\right)$

$$
\begin{aligned}
& u_{i=i \max }=v_{i=i \max }=0, \quad p_{i=i \max }=p_{\infty, i=i \max }, \\
& T_{i=i \max }=T_{\infty, i=i \max }
\end{aligned}
$$

- Slip wall condition on the ground $(h=0 \mathrm{~km})$

$$
\begin{gathered}
u_{i=i g}=0, \quad v_{i=i g}=2 v_{i=i g-1}-v_{i=i g-2} \\
p_{i=i g}=p_{0}+\Delta p_{i=i g-1}, \quad T_{i=i g}=T_{0}+\Delta T_{i=i g-1}
\end{gathered}
$$

where the subscripts $i$ and $j$ denote the indexes of grid points in the $R$ and $\theta$ directions, respectively, and the subscript $i g$ denotes the index of grid points on the ground. 


\section{Numerical Results}

\subsection{Blast wave in uniform atmosphere}

The pressure rise of a blast wave produced by spherical explosives in a uniform atmosphere can be estimated using the empirical formulas that were derived from the experimental and computational approaches. ${ }^{7,8)}$ In this study, computational accuracy in the uniform atmosphere was validated by comparison with the results obtained applying Brode's formula $^{8)}$ as Eqs. (14) and (15), which is one of the most famous TNT-equivalent methods.

$$
\begin{gathered}
\Delta p_{s}=\frac{670}{Z^{3}}+100, \quad(\Delta p>1,000) \\
\Delta p_{s}=\frac{97.5}{Z}+\frac{145.5}{Z^{2}}+\frac{585}{Z^{3}}-1.9, \quad(10<\Delta p<1,000) \\
Z=\frac{R}{E_{\mathrm{TNT}}{ }^{1 / 3}}
\end{gathered}
$$

where the unit of $\Delta p_{s}$ is $\mathrm{kPa}$ and that of $Z$ is $\mathrm{m} / \mathrm{kg}^{1 / 3}$. In Brode's formula, the point explosion in a uniform atmosphere at the ground is assumed. The propagation speed of the blast wave is calculated using the Rankine-Hugoniot relation $^{22)}$ :

$$
u_{s}=c_{\infty} \sqrt{1+\frac{\gamma+1}{2 \gamma} \frac{\Delta p_{s}}{p_{\infty}}}
$$

In addition, the propagation distance of the shock wave is calculated by determining the integral of the propagation speed over the propagation time. In the simulation, the core radius was set to $R_{\text {core }}=50$ and $100 \mathrm{~m}$, and the uniform properties of the atmosphere, where $T_{\infty}=288.15 \mathrm{~K}$ and $p_{\infty}=101.325 \mathrm{kPa}$, was assumed. The other conditions were the same as the conditions of the Chelyabinsk meteorite (see Sec. 2.1 for details). Figure 4 shows the comparison between the simulation results and the results of Brode's formula. In this figure, only the simulation result for $R_{\text {core }}=100 \mathrm{~m}$ is described because the pressure rise was almost the same regardless of the core radius. As shown in Fig. 4, the simulation results agree well with the results of Brode's formula. Thus, it can be seen that direct simulation is applicable for the unsteady analysis of blast waves.

\subsection{Chelyabinsk meteorite}

The Chelyabinsk meteorite ${ }^{3,4)}$ was observed in many videos and observation equipment, and the observational data is useful for validating the computational accuracy when considering actual atmospheric conditions. Thus, computations were made to reproduce the blast wave generated from the Chelyabinsk meteorite as described in Chap. 2. An analysis of the simulation results obtained using the fine grids is presented in this section, because the grid convergence was confirmed in Section 3.2.

\subsubsection{Pressure and temperature fluctuation}

Figure 5 shows the distribution of time series variation in pressure and temperature fluctuations from the atmospheric properties, which are described by considering symmetry

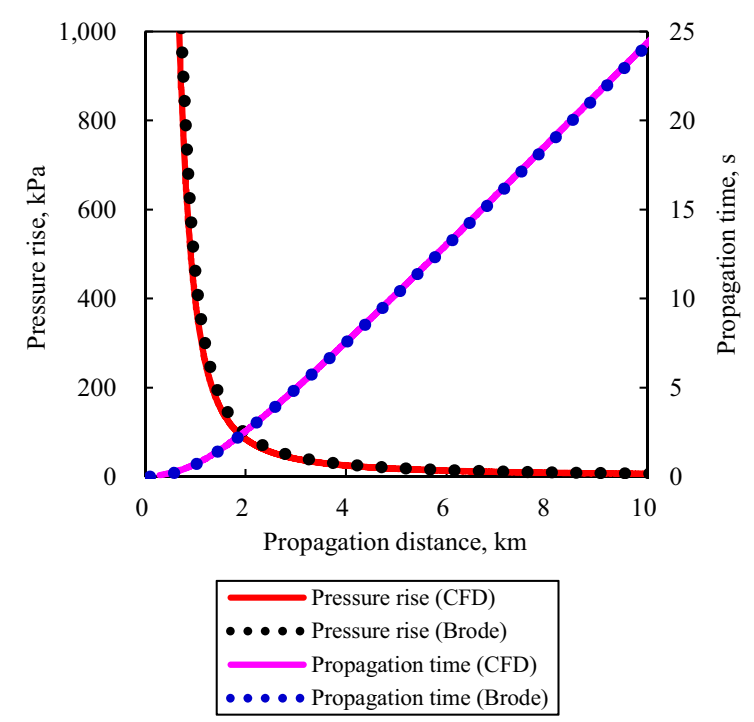

Fig. 4. Comparison between simulation results obtained using the fine grid and Brode's empirical formula.

with respect to the vertical $x$-axis. In the close-up figure of temperature fluctuation, the velocity vector is depicted. Propagation of the blast wave can be investigated using the distribution of pressure fluctuation as follows: As shown in Fig. 5(a), the primary shock wave propagates spherically from the spherical core, and a secondary shock wave is generated in the over-expanded region behind the primary shock wave (see Section 4.2.2 for details). As shown in Fig. 5(b), the nature of blast wave propagation is changed according to the propagation direction because of variation in the atmospheric properties with altitude. As shown in Fig. 5(c), regular reflection of the waves occurs on the ground, and the reflected waves propagate spherically upward. The pressure fluctuation is increased in the region where the incident and reflected shock waves interact with each other, whereas it is decreased in the region where the expansion waves behind the primary shock wave interact with other waves. As shown in Fig. 5(d), the location of the peak pressure rise moves away from ground zero as the propagation time of the waves lengthens.

The behavior of hot air in the core can be investigated by analyzing the distribution of temperature fluctuation and velocity vector as follows: As shown in Figs. 5(a) and 5(b), the hot air rises due to the buoyancy effects and the vertical velocity decreases as the distance from the vertical $x$-axis increases. The vortex structures around the hot air are generated as a result of the vertical movement of hot air, as shown in Figs. 5(c) and 5(d). As a result, the spherical core is changed to a shape like a mushroom cloud. Since the velocity of hot air in the core is much slower than the propagation speed of the blast wave, the behavior of the hot air including the vortex phenomenon hardly affects the strength of the blast wave on the ground.

\subsubsection{Pressure waveform}

Figure 6 shows the pressure waveform on the axis of symmetry under the spherical core in a standard atmosphere. At $t=0-0.4 \mathrm{~s}$, the primary shock wave is generated from the 
Trans. Japan Soc. Aero. Space Sci., Vol. 61, No. 3, 2018
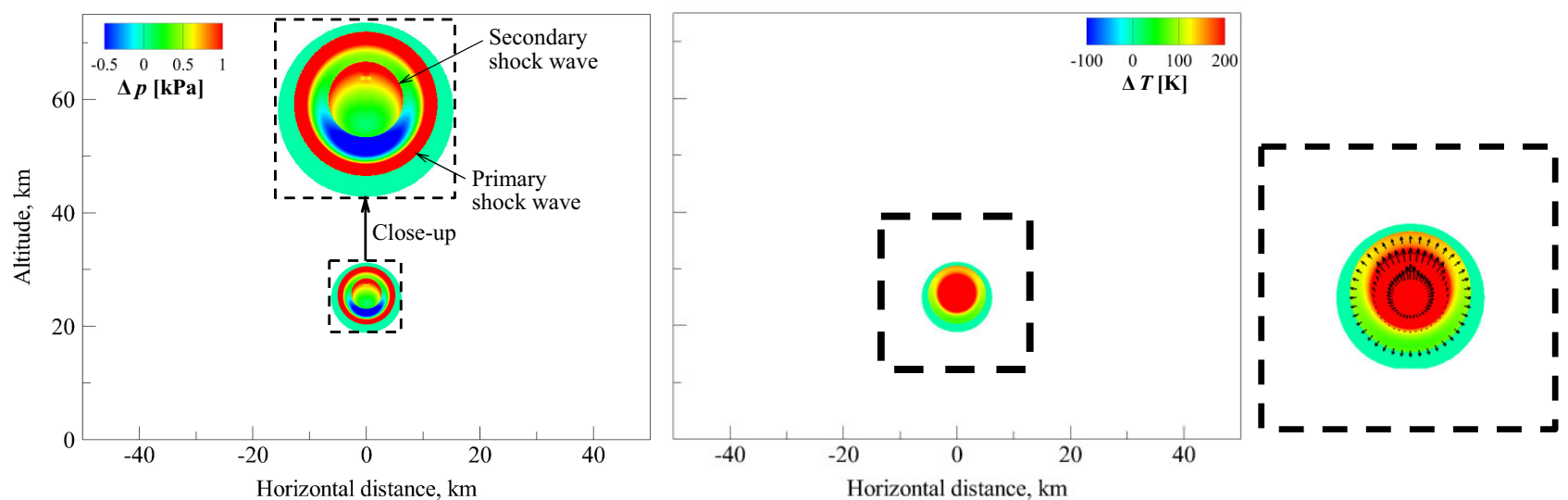

(a) $t=5 \mathrm{~s}$
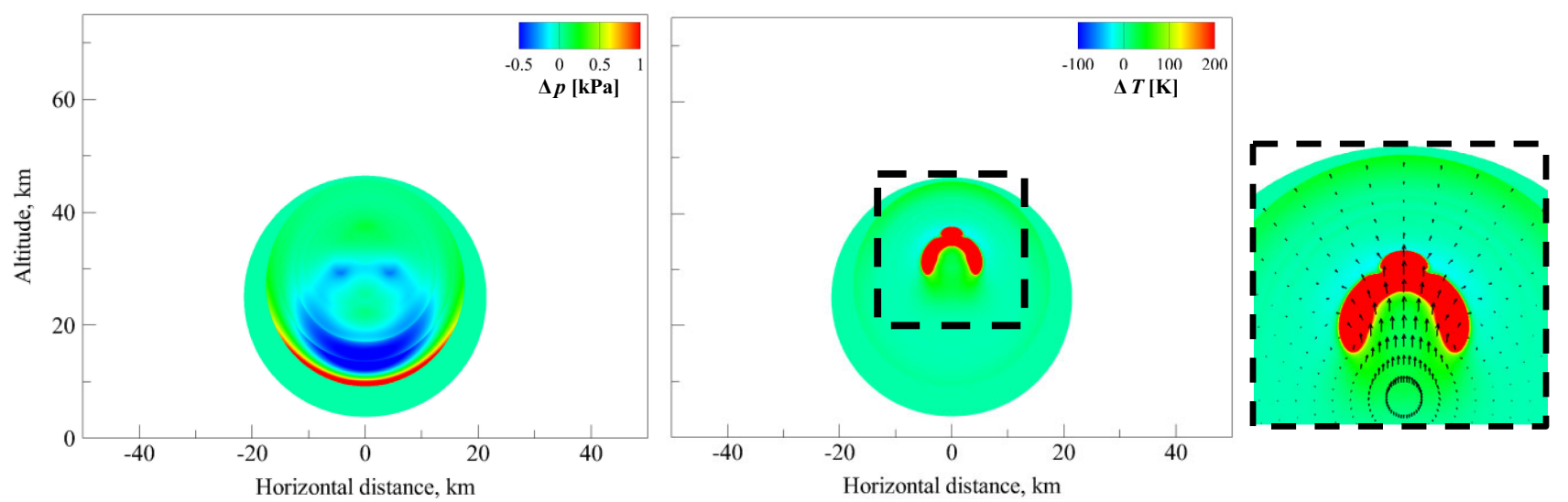

(b) $t=40 \mathrm{~s}$
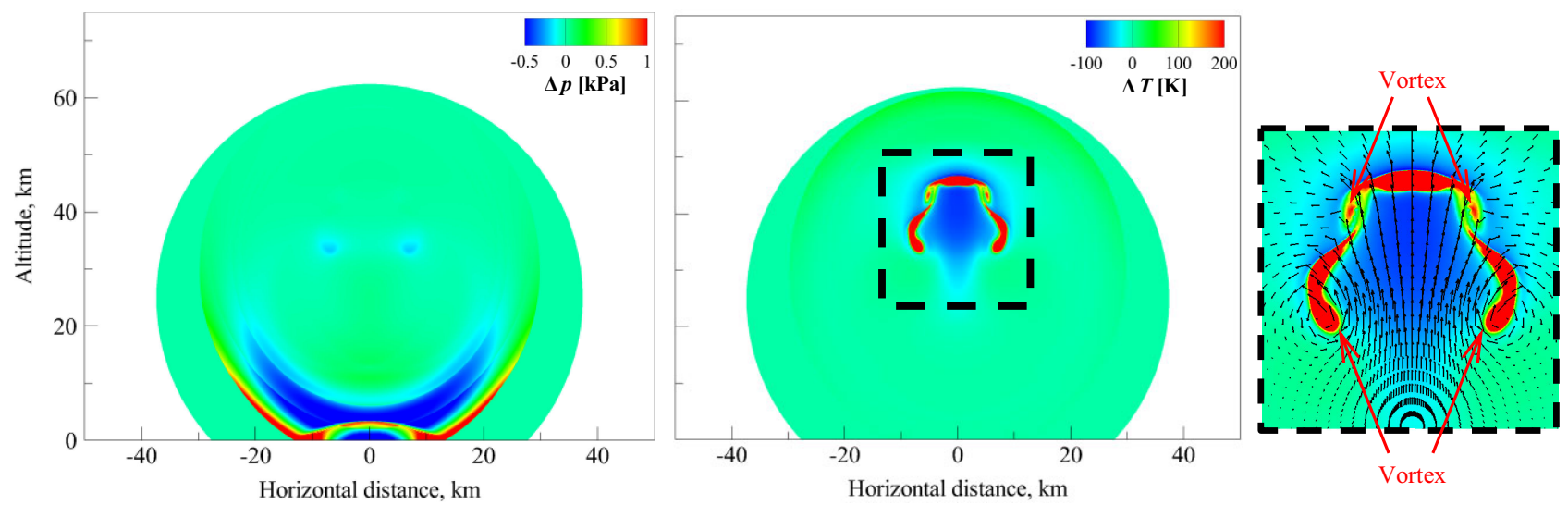

(c) $t=80 \mathrm{~s}$
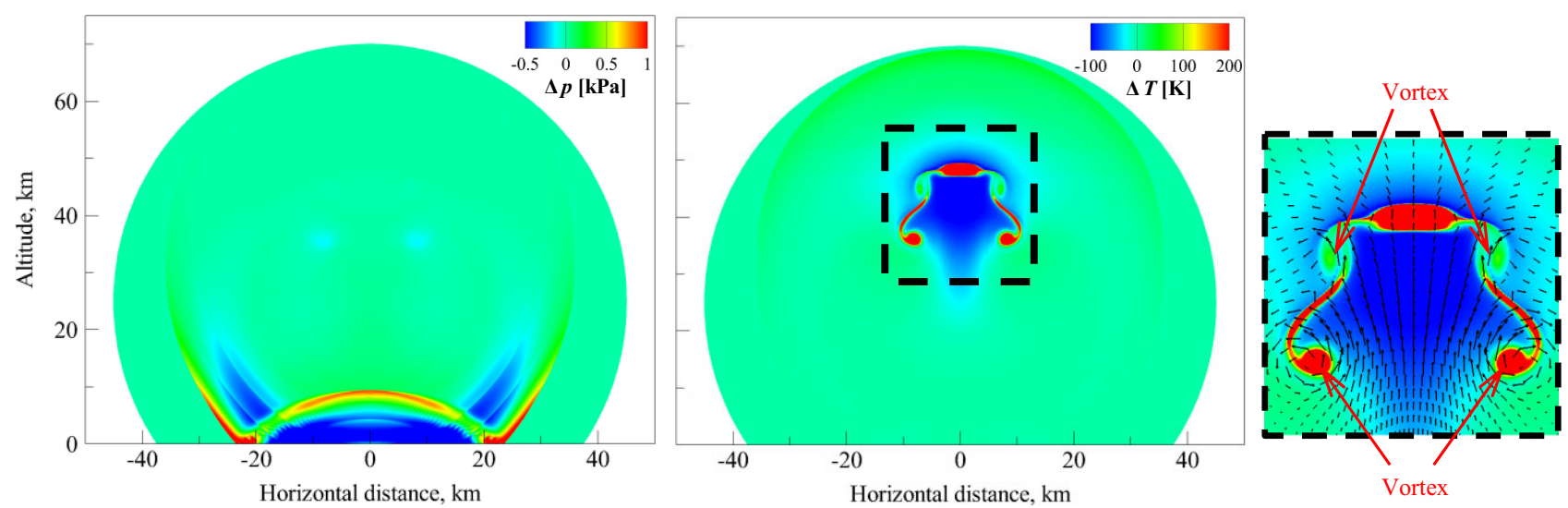

(d) $t=100 \mathrm{~s}$

Fig. 5. Pressure (left) and temperature (center and right) fluctuations from atmospheric properties in a standard atmosphere. 


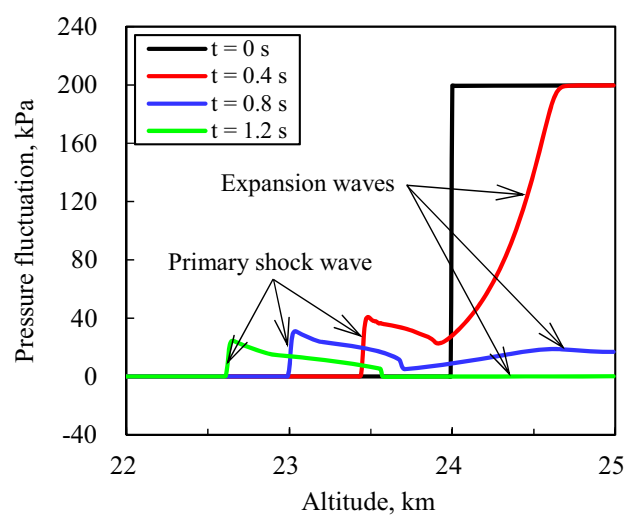

(a) $t=0,0.4,0.8,1.2 \mathrm{~s}$

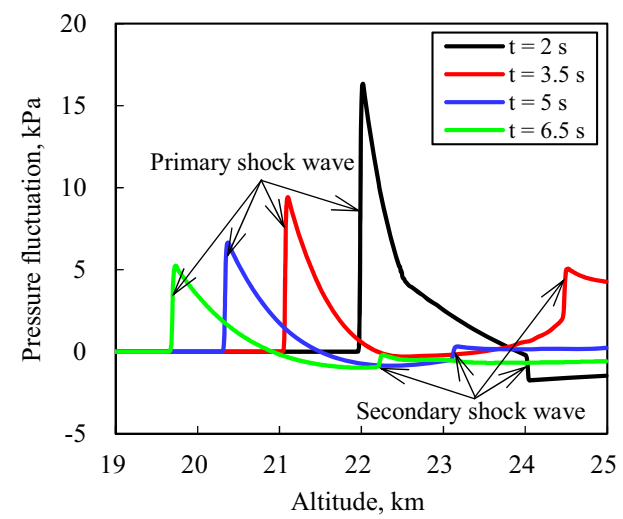

(b) $t=2,3.5,5,6.5 \mathrm{~s}$

Fig. 6. Pressure waveform on the axis of symmetry under the spherical core in a standard atmosphere.

surface of the spherical core and expansion waves are generated in the core, as shown in Fig. 6(a). At $t=0.4-1.2 \mathrm{~s}$, these expansion waves propagate toward the center of the explosion and are reflected. Consequently, the pressure behind the primary shock wave is significantly decreased and the over-expanded region is generated. Then, the ambient gas flowing into the over-expanded region converges and the secondary shock wave is generated at $t=1.2-2 \mathrm{~s}$, as shown in Fig. 6(b). At $t=2-3.5 \mathrm{~s}$, the secondary shock wave propagates toward the center of the explosion and is reflected. Thereafter, the reflected secondary shock wave follows the primary shock wave.

Figure 7 shows the pressure waveforms at ground zero. Note that the pressure fluctuation was doubled by the reflection of the waves on the ground. As shown in Fig. 7, the configuration of the pressure waveform is almost the same as that at $t=6.5 \mathrm{~s}$ shown in Fig. 6(b). This is because the propagation speed of the primary shock wave is faster than that of the secondary shock wave, and the two shock waves do not interact with each other. The pressure waveform in a uniform atmosphere is quite different from that in another form of atmosphere. The results show that the change in atmospheric properties with altitude must be considered to analyze the blast wave generated from a meteorite explosion at high altitude. The pressure waveform in an isothermal atmosphere is almost the same as that in a standard atmosphere. However,

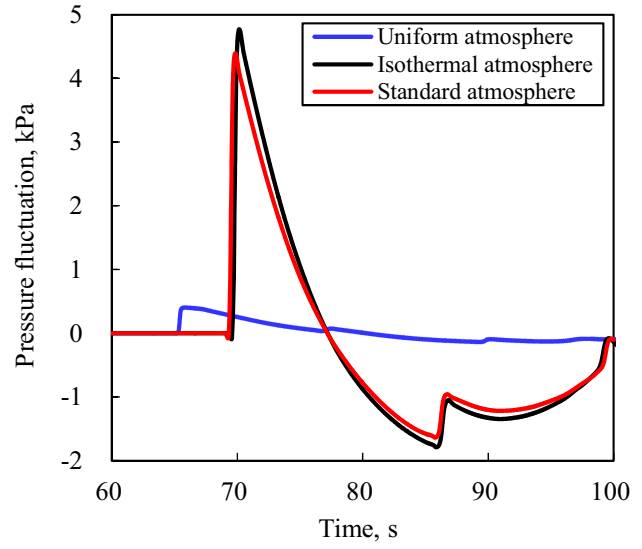

Fig. 7. Comparison of pressure waveforms at ground zero.

the pressure rise of the primary shock waves differs from each other. Therefore, it can be seen that a standard atmosphere should be considered to accurately evaluate the blast wave strength.

\subsubsection{Pressure rise and arrival time}

Figures 8 and 9 show the pressure rise of the primary shock wave and its propagation distance on the axis of symmetry under the spherical core, respectively. As shown in Fig. 8, the pressure rise in the vicinity of the spherical core decreases rapidly because of the geometrical spreading of the primary shock wave from the center of the explosion. The rate of decrease in pressure rise differs according to the atmospheric model used. Since the atmospheric pressure in isothermal and standard atmospheres increases toward the ground, as shown in Fig. 2, the pressure rise decreases and increases toward the ground, although in a uniform atmosphere it decreases monotonically. The pressure rise in a standard atmosphere reaches the minimum value at an altitude of $8.2 \mathrm{~km}$ and increases by $5.3 \%$ before reaching the ground, whereas that in an isothermal atmosphere reaches the minimum value at an altitude of $9.7 \mathrm{~km}$ and increases by $23.1 \%$ before reaching the ground. At low altitudes, the rate of change in atmospheric pressure in an isothermal atmosphere is higher than that in a standard atmosphere, as shown in Fig. 2. Therefore, the rate of change in pressure rise in an isothermal atmosphere is higher than that in a standard atmosphere, as shown in Fig. 8. As shown in Fig. 9, the propagation distance of the primary shock wave, except in the vicinity of the spherical core, increases linearly because the blast wave decays as the propagation distance increases and the propagation speed in the region far from the center of the explosion converges into the speed of sound; that is, there is virtually no change in propagation speed in uniform and isothermal atmospheres, whereas the change in a standard atmosphere is based on variation in atmospheric temperature with altitude. The propagation distance in a standard atmosphere was almost the same as that in an isothermal atmosphere: the average speed of sound at $h=0$ to $25 \mathrm{~km}$ in a standard atmosphere is almost the same as the speed of sound in an isothermal atmosphere. The propagation speed in a uniform atmosphere is faster than those in isother- 


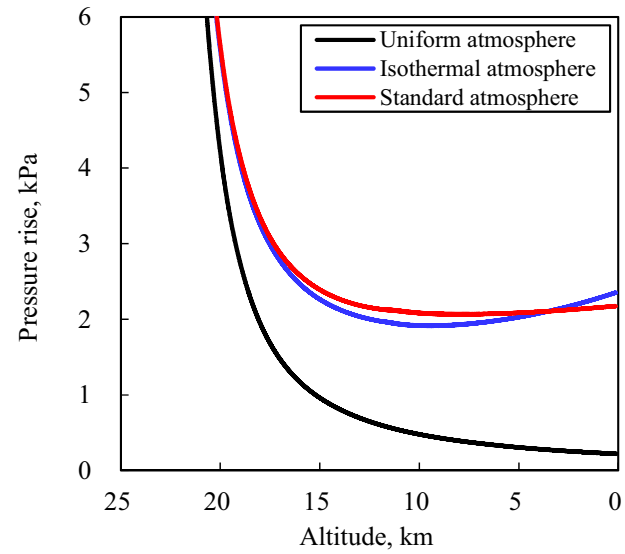

Fig. 8. Variation in pressure rise on the axis of symmetry under the spherical core.

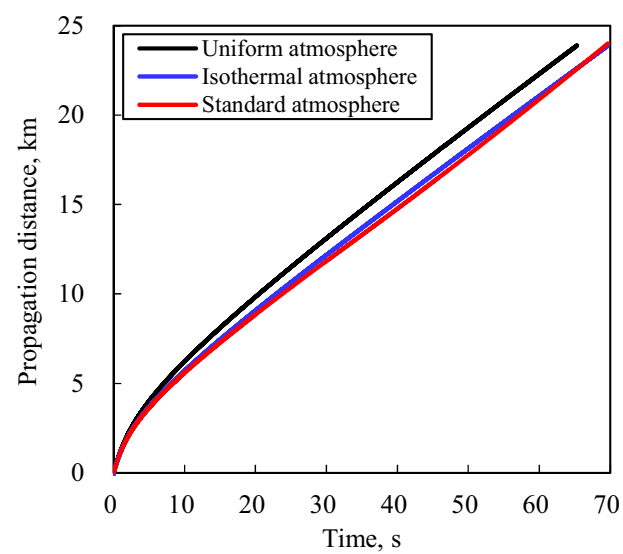

Fig. 9. Propagation distance of primary shock wave from the surface of the spherical core on the axis of symmetry under the core.

mal and standard atmospheres before converging into the speed of sound. This is because, according to the RankineHugoniot relation given by Eq. (17), the propagation speed depends on $\Delta p_{s} / p_{\infty}$ and $c_{\infty} . \Delta p_{s} / p_{\infty}$ in a uniform atmosphere is higher than those in isothermal and standard atmospheres, although $c_{\infty}$ was a bit slower in a uniform atmosphere. As a result, the propagation speed calculated using Eq. (17) for a uniform atmosphere was faster than those of isothermal and standard atmospheres before converging into the speed of sound. These results show that the characteristics of blast wave propagation through uniform, isothermal, and standard atmospheres were well clarified by direct simulation.

Figures 10 and 11 show the variation in pressure rise and arrival time at ground-level, respectively. Although the numerical oscillation of pressure rise near the axis of symmetry is confirmed, this oscillation was on the same order of the grid convergence (see Section 3.2 for details); thus, the influence of oscillation seems to have very little effect on the pressure rise. As shown in Fig. 10, the pressure rise decreases as the distance from ground zero increases, regardless of the difference in atmospheric models. The pressure rise in an isothermal atmosphere relative to that in a standard atmosphere increases by $8.9 \%$ at $r=0 \mathrm{~km}$ and $7.1 \%$ at $r=40 \mathrm{~km}$. This is

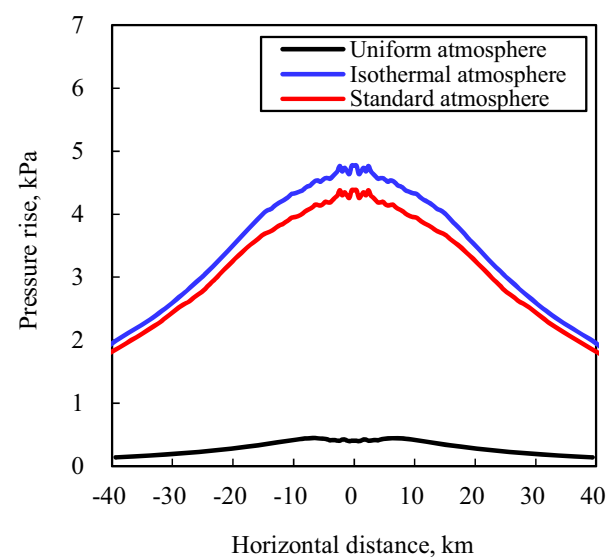

Fig. 10. Variation in pressure rise at ground-level.

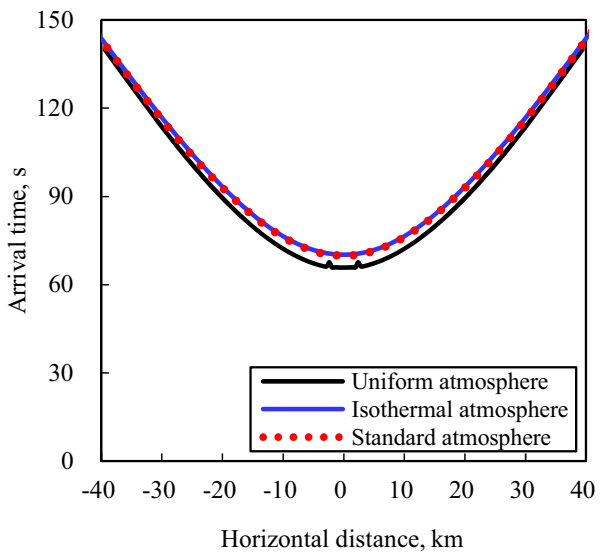

Fig. 11. Variation in arrival time at ground-level.

because the rate of change in atmospheric pressure is different in isothermal and standard atmospheres, as shown in Fig. 2. On the other hand, the arrival time in an isothermal atmosphere is almost the same as that in a standard atmosphere, as shown in Fig. 11. This is because the average of speed of sound as a function of atmospheric temperature is almost the same for isothermal and standard atmospheres. These results show that the pressure rise depends on the rate of change in atmospheric pressure, whereas the arrival time does not depend on the rate of change in atmospheric temperature. Therefore, it can be seen that a standard atmosphere must be considered to accurately evaluate blast wave strength.

The analysis of observational data from the Chelyabinsk meteorite confirmed that the pressure rise of the blast wave estimated from data on window breakage was 0.7 to $3.8 \mathrm{kPa}^{3,4)}$ at the center of Chelyabinsk, i.e., a distance of approximately $40 \mathrm{~km}$ from ground zero. The arrival time that it took the explosive sound to reach the center of Chelyabinsk after the peak brightness was 140 to $146 \mathrm{~s} .{ }^{6,15)}$ As shown in Figs. 10 and 11, the pressure rise obtained as the result of direct simulation in a standard atmosphere was $1.8 \mathrm{kPa}$ at $r=40 \mathrm{~km}$, and the arrival time was $143 \mathrm{~s}$ at $r=40 \mathrm{~km}$. These results demonstrate that direct simulation considering 
a standard atmosphere successfully reproduced a blast wave strength that is strong enough to cause serious damage, ${ }^{23)}$ as observed in Chelyabinsk. Therefore, it can be said that direct simulation considering a stratified atmosphere is a powerful tool for the unsteady analysis of blast waves generated from meteorite explosions.

\section{Conclusions}

This paper presented a method for directly simulating the blast wave generated at the time of a meteorite explosion. The simulation results in a uniform atmosphere were in good agreement with Brode's empirical formula, which is one of the most famous TNT-equivalent methods. The simulation considering a standard atmosphere successfully reproduced the blast wave generated by the meteorite explosion during the Chelyabinsk event. Moreover, the characteristics of blast wave propagation through uniform, isothermal, and standard atmospheres were well clarified using the simulation. Consequently, it was shown that a standard atmosphere must be considered to accurately evaluate the blast wave generated from meteorite explosions at high altitude. These results demonstrate the usefulness of this simulation as an approach for the unsteady analysis of blast wave propagation through a stratified atmosphere, which is required for the risk management of meteorite fall.

\section{Acknowledgments}

The first author was supported by Grant-in-Aid for Scientific Research No. 15J06433 presented by the Japan Society for the Promotion of Science.

\section{References}

1) Takahashi, N.: The Natural Disaster Risk by Near Earth Asteroids -Observation, Risk Assessment and Measures-, Aeronaut. Space Sci. Japan, 65 (2017), pp. 123-128 (in Japanese).

2) Shuvalov, V. V., Svettsov, V. V., and Trubetskaya, I. A.: An Estimate for the Size of the Area of Damage on the Earth's Surface after Impacts of 10-300-m Asteroids, Solar System Research, 47 (2013), pp. 260-267.

3) Brown, P. G., Assink, J. D., Astiz, L., Blaauw, R., Boslough, M. B., Borovička, J., Brachet, N., Brown, D., Campbell-Brown, M., Geranna, L., Cooke, W., de Groot-Hedlin, C., Drob, D. P., Edwards, W., Evers, L. G., Garces, M., Gill, J., Hedlin, M., Kingery, A., Laske, G., Le Pichon, A., Mialle, P., Moser, D. E., Saffer, A., Silber, E., Smets, P., Spalding, R. E., Spurný, P., Tagliaferri, E., Uren, D., Weryk, R. J., Whitaker, R., and Krzeminski, Z.: A 500-Kiloton Airburst over Chelyabinsk and an Enhanced Hazard from Small Impac- tors, Nature, 503 (2013), pp. 238-241.

4) Emel'yanenko, V. V., Popova, O. P., Chugai, N. N., Shelyakov, M. A., Pakhomov, Yu. V., Shustov, B. M., Shuvalov, V. V., Biryukov, E. E., Rybnov, Yu. S., Marov, M. Ya., Rykhlova, L. V., Naroenkov, S. A., Kartashova, A. P., Kharlamov, V. A., and Trubetskaya, I. A.: Astronomical and Physical Aspects of the Chelyabinsk Event (February 15, 2013), Solar System Res., 47 (2013), pp. 240-254.

5) Vasilyev, N. V.: The Tunguska Meteorite Problem Today, Planetary Space Sci., 46 (1998), pp. 129-150.

6) Aftosmis, M. J., Nemec, M., Mathias, D. L., and Berger, M. J.: Numerical Simulation of Bolide Entry with Ground Footprint Prediction, AIAA 2016-0998, 2016.

7) Glasstone, S. and Dolan, P. J.: The Effects of Nuclear Weapons, Third Ed., U. S. Government Printing Office, Washington, 1977.

8) Brode, H. L.: Numerical Solutions of Spherical Blast Waves, J. Appl. Phys., 26 (1955), pp. 766-775.

9) Alonso, J. J. and Colonno, M. R.: Multidisciplinary Optimization with Applications to Sonic-Boom Minimization, Annu. Rev. Fluid Mech., 44 (2012), pp. 505-526.

10) Thomas, C. L.: Extrapolation of Sonic Boom Pressure Signatures by the Waveform Parameter Method, NASA TN D-6832, 1972.

11) Whitham, G. B.: The Flow Pattern of a Supersonic Projectile, Commun. Pure Appl. Mathematics, 5 (1952), pp. 301-348.

12) Saito, T., Kitamura, T., Takayama, K., Fujii, N., and Taniguchi, H.: Numerical Simulations of Blast Wave Propagation Induced by Eruptions of Volcanoes, Shock Waves Marseille, III (1995), pp 385-390.

13) Fujii, K., Shimizu, F., Tamura, Y., Higashino, F., Hinada, M., and Akiba, R.: Blast Wave Simulation with Ground Surface Effect, Proceedings of the 17th International Symposium on Space Technology and Science, 1990, pp. 759-764.

14) Middha, P. and Hansen, O. R.: Using Computational Fluid Dynamics as a Tool for Hydrogen Safety Studies, J. Loss Prevention Process Industries, 22 (2009), pp. 295-302.

15) Avramenko, M. I., Glazyrin, I. V., Ionov, G. V., and Karpeev, A. V.: Simulation of the Airwave Caused by the Chelyabinsk Superbolid, J. Geophys. Res. Atmosphere, 119 (2014), pp. 7035-7050.

16) Yamashita, R. and Suzuki, K.: Full-Field Simulation for Sonic Boom Cutoff Phenomena, Trans. Jpn. Soc. Aeronaut. Space Sci., 58 (2015), pp. 327-336.

17) Yamashita, R. and Suzuki, K.: Full-Field Sonic Boom Simulation in Stratified Atmosphere, AIAA J., 54 (2016), pp. 3223-3231.

18) ISO 2533, 1975.

19) Wada, Y. and Liou, M. S.: A Flux Splitting Scheme with High-Resolution and Robustness for Discontinuities, AIAA Paper 94-0083, 1994.

20) van Leer, B.: Towards the Ultimate Conservative Difference Scheme. IV. A New Approach to Numerical Convection, J. Comput. Phys., 23 (1977), pp. 276-299.

21) van Albada, G. D., van Leer, B., and Roberts, W. W., Jr.: A Comparative Study of Computational Methods in Cosmic Gas Dynamics, Astronomy Astrophysics, 108 (1982), pp. 76-84.

22) Liepmann, H. W. and Roshko, A.: Elements of Gas Dynamics, John Wiley and Sons, New York, 1957.

23) Takayama, K.: Shock Wave Handbook, Springer Verlag, Tokyo, 1995 (in Japanese). 